\title{
Activation of neutrophils by a novel triggering immunoglobulin-like receptor MAIR-IV
}

\author{
Takako Nakano, Satoko Tahara-Hanaoka, Chigusa Nakahashi, Ismail Can, \\ Naoya Totsuka, Shin-ichiro Honda, Kazuko Shibuya, and Akira Shibuya ${ }^{1}$
}

Department of Immunology, Institute of Basic Medical Sciences and Center for TARA, Graduate School of Comprehensive Human Sciences, University of Tsukuba, Ibaraki 305-8575, Japan.

${ }^{1}$ Correspondence should be addressed to Akira Shibuya, M.D., Ph.D.

Department of Immunology, Institute of Basic Medical Sciences, Graduate School of Comprehensive Human Sciences, University of Tsukuba, 1-1-1, Ten-nodai, Tsukuba, Ibaraki 305-3585, Japan (e-mail; ashibuya@md.tsukuba.ac.jp, FAX: 81-29-853-3410, Phone: 81-29-853-3474) 
Abstract

Neutrophils play a central role in host defenses against infectious microbial pathogens.

However, molecular basis for their triggering signals has been incompletely understood.

Here, we show molecular and functional characteristics of myeloid-associated

immunoglobulin-like receptor (MAIR)-IV. MAIR-IV was preferentially expressed on

neutrophils in the peripheral blood, bone marrow, peritoneal cavity and spleen.

MAIR-IV physically associates with immunoreceptor tyrosine based activating motif

(ITAM)-bearing adaptor FcR $\gamma$ chain. Cross-linking MAIR-IV with anti-MAIR-IV

monoclonal antibody significantly induced secretion of proinflammatory cytokines

TNF- $\alpha$ and IL-6 from neutrophils. Thus, MAIR-IV may regulate activation of neutrophils and play an important role for innate immunity.

\section{Key Words}

Neutrophils, MAIR-IV, CLM5, FcR $\gamma$ chain, innate immunity 


\section{Introduction}

Myeloid cells, including neutrophils and macrophages, play an essential role for host

defenses against infectious microbial pathogens (Nathan, 2006). In response to

inflammatory stimuli, neutrophils are activated through a vast array of cell surface

receptors, such as Fc receptors for IgG, $\beta 2$ integrins, complement receptors, chemokines,

cytokine receptors and Toll-like receptors, and mediate phagocytosis, degranulation of

anti-microbial substances or secretion of inflammatory mediators. However, the

molecular interactions that trigger anti-microbial functions of neutrophils are

incompletely understood.

We have recently identified paired activating and inhibitory receptors,

myeloid-associated immunoglobulin (Ig)-like receptor (MAIR)-I and MAIR-II

(Yotsumoto et al., 2003), which are also named as CLM8/LMIR1 and

CLM4/LMIR2/DIgR1, respectively (Luo et al., 2001)(Kumagai et al., 2003)(Chung et

al., 2003). MAIR-I is preferentially expressed on myeloid cells, including 
macrophages, neutrophils, dendritic cells (DC) and bone marrow-derived cultured mast

cells (Yotsumoto et al., 2003) and mediates an inhibitory signal through

immunoreceptor tyrosine-based inhibitory motif (ITIM) in the cytoplasmic portion.

On the other hand, MAIR-II, expressed on macrophages, associates with either immunoreceptor tyrosine-based activating motif (ITAM)-containing adaptors DAP12 or FcR $\gamma$ chain and mediates an activating signal, resulting in inflammatory cytokine secretion from macrophages (Yotsumoto et al., 2003)(Nakahashi et al., 2007). By screening a database for the mouse genome, we and others found that MAIR-I and MAIR-II are members of a multigene family consisting of nine genes on a small segment of mouse chromosome 11 (our unpublished observation and (Chung et al., 2003)).

In this study, we generated monoclonal antibodies (mAbs) against a member of the family, MAIR-IV, and investigated its cellular distribution and functional role in immune responses. We show that MAIR-IV is a novel triggering receptor on neutrophils and is involved in inflammatory responses. 


\section{Materials and methods}

\subsection{Antibodies}

Anti-MAIR-IV mAbs TX-69 and TX81 (rat IgG2a and IgG2b, respectively) were generated and $\mathrm{F}(\mathrm{ab})_{2}$ fragments of TX-69 mAb were prepared in our laboratory, as described (Yotsumoto et al., 2003). Alexa Fluor 647-conjugated F(ab’) $)_{2}$ fragments of TX69 was prepared by using Alexa Fluor 647 monoclonal antibody labeling kit (Molecular Probes, Eugene, Oregon). Control rat and mouse IgG, anti-CD11b (Mac-1), anti-Ly6G (Gr-1), anti-B220, anti-CD3, anti-CD11c, DX-5, and anti-CD32/16 (2.4G2) mAbs were purchased from BD Biosciences (San Jose, CA). Anti-Flag mAb, anti-FceRI $\gamma$ (FcR $\gamma$ chain) mAb and horse-radish peroxidase (HRP)-conjugated streptavidin (SA) were purchased from Sigma-Aldrich (St Louis, MO), Upstate Biotechnology (Lake Placid, NY) and Amersham Biosciences (Piscataway, NJ), respectively. To exclude endotoxin contamination, all antibodies were treated with Polymyxin B (BIO-RAD, CA), resulting in endotoxin contamination at less than 100 pg/mg of mAb, as determined by Limulus Color KY Test (Wako, Osaka, Japan). 
Nakano T, et al

\subsection{Mice, Cells and transfectants}

FcR $\gamma^{-/-}$mice were provided by T. Takai (Tohoku University, Sendai, Japan). C57BL/6J

mice were purchased from Clea Japan (Tokyo, Japan). Neutrophils from the bone

marrow were purified by using a MACS selection system using biotin-labeled anti-Gr-1,

followed by streptavidin-MicroBeads (Miltenyi Biotec, Bergisch Granbach, Germany)

and those from peritoneal cavity were collected 6 hrs after the 2.4\% thioglycollate i.p.

injection. Macrophages, dendritic cells (DC) and mast cells were generated by culture of BM cells in the presence of M-CSF (50 ng/ml) for 5 days, GM-CSF (10 ng/ml) for 9 days and IL-3 (4 ng/ml) plus SCF (10 ng/ml) for 5 weeks, respectively. Purity of each lineage of cells was more than 95\%, as determined by flow cytometry. All experiments were performed according to the guidelines of the animal ethics committee of the University of Tsukuba. BW5147, RAW264.7 and RBL-2H3 transfectants stably expressing MAIR-IV, MAIR-II or MAIR-V were established, as described (Yotsumoto et al., 2003). 


\subsection{Immunoprecipitation and western blotting}

To analyze association of MAIR-IV with FcR $\gamma$ chain, RAW264 or RBL-2H3 transfectants, or peritoneal exudative macrophages were lysed with a lysis buffer containing $1 \%$ digitonin (Calbiochem, San Diego, CA), $\quad 0.12 \%$ Triton-X (Sigma-Aldrich), $150 \mathrm{mM} \mathrm{NaCl}, 20 \mathrm{mM}$ triethanolamine and protease inhibitors. Lysates were subjected to immunoprecipitation with anti-Flag M2 affinity gel (Sigma Aldrich), anti-MAIR-IV mAb (TX81) or isotype-matched rat IgG. Immunoprecipitates were separated by SDS-PAGE under reducing condition, transferred (60V in 25mM Tris, 195mM glycine, and 20\% methanol) to PVDF membranes (Immobilon-PSQ; Millipore, Billerica, MA). Membranes were incubated overnight in TBST (10mM Tris-buffered saline containing $0.5 \%$ Tween 20, 0.5g/l MgCl2, pH8.0), containing 3\% bovine serum albumin (BSA) for blocking, and incubated with primary antibodies. Proteins were detected by using HRP-conjugated anti-rabbit IgGs or anti-rat IgGs (Amersham Biosciences, Piscataway, NJ), and were developed with SuperSignal CL-HRP substrate (Piece Chemical Co.). For stripping and re-blotting, Restore Western Blot Stripping Buffer (Piece Chemical Co.) was used according to the manufacture’s instruction. 
Nakano T, et al

2.4. Cytokine production

Peritoneal exudative cells (PECs) and neutrophils were stimulated with plate-coated

$\mathrm{F}\left(\mathrm{ab}^{\prime}\right)_{2}$ fragments of anti-MAIR-IV mAb (TX69) or control IgG and culture

supernatants were subjected to ELISA assay for TNF- $\alpha$ and IL-6 (BD Biosciences, San

Jose, CA). 


\section{Results and discussion}

\subsection{Identification of MAIR-IV}

We cloned all the full-length cDNAs of the MAIR family genes other than MAIR-I and MAIR-II by PCR from the spleen of C57BL/6 mice and designated them as MAIR-III to MAIR-IX, based on a phylogenetic tree analysis (Fig1A, B). The MAIR family was found to be a murine counterpart of the human CMRF-35 (CD300) family (Cantoni et al., 1999)(Clark et al., 2000)(Clark et al., 2002), which is located on human chromosome 17, syntenic region of mouse chromosome 11 (Fig 1A). We found that one of the genes, MAIR-IV, has a short cytoplasmic tail (24 aa) with no signaling motif and a negatively charged glutamic acid (E) in its transmembrane (TM) region. The Ig-like domain of MAIR-IV in the extracellular portion has 91\% identity with that of MAIR-V at the amino acid level. In contrast to MAIR-IV, MAIR-V has a long cytoplasmic tail containing two consensus immunoreceptor tyrosine-based inhibitory motif (ITIM), suggesting that MAIR-IV and MAIR-V constitute paired activating and inhibitory receptors. 
3.2. MAIR-IV is preferentially expressed on neutrophils and macrophages

MAIR-IV and MAIR-V were also named as CLM5 and CLM1/DIgR2 (Chung et al., 2003)(Fujimoto et al., 2006)(Shi et al., 2006), respectively. Although expression of MAIR-IV/CLM5 mRNA was reported to be detected in myeloid cells (Fujimoto et al., 2006), its protein expression profile has been undetermined because of lack of monoclonal antibodies. Therefore, functional characteristics of MAIR-IV in immune responses has remained unclear. Here, we generated monoclonal antibodies (TX69 and TX81 mAbs) against MAIR-IV by immunizing a rat with a mouse T cell leukemia cell line BW5147 transfected with Flag-tagged MAIR-IV. To confirm the specificity of these mAbs to MAIR-IV, we also established BW5147 transfectant stably expressing MAIR-I, MAIR-II, MAIR-V tagged with Flag at the N-terminus. TX69 and TX81 mAbs specifically stained the transfectant expressing MAIR-IV, but not those expressing MAIR-I, MAIR-II or MAIR-V, indicating that these mAbs are specific to MAIR-IV (Fig 2A and data not shown). Analyses by flow cytometry by using Alexa-657-conjugated F(ab’) 2 fragments of TX69 mAb showed that MAIR-IV was 
preferentially expressed on Gr- $1^{\text {high }} / \mathrm{Mac}-1^{+}$neutrophils from the peripheral blood, bone marrow, peritoneal cavity and spleen (Fig.2B). MAIR-IV was also expressed on Mac $-1^{+} / \mathrm{Gr}-1^{-}$macrophages from the spleen and bone marrow and $\mathrm{CD} 11 \mathrm{c}^{+}$dendritic cells (DC), but not on T, B, NK cells or bone marrow-derived cultured mast cells (Fig.2B).

3.3. FCR $\gamma$ chain physically associates with MAIR-IV and is involved in cell surface expression of MAIR-IV in primary cells

MAIR-IV contains a short cytoplasmic tail with no signaling motif, suggesting that

MAIR-IV, like MAIR-II, mediates activating signals via ITAM-containing adapter proteins, such as FcR $\gamma$ chain or DAP12. These adaptor proteins are expressed in myeloid cells and characterized by a small extracellular region, a negatively charged amino acid aspartic acid (D) in the TM domain, and a cytoplasmic domain that contains two ITAMs (Feng et al., 2005). DAP12 associates with several activating NK receptors and myeloid cell-specific receptors, including human and mouse TREM-1, TREM-2, MDL-1, and PILR $\beta$, SIRP $\beta 1$, mouse TREM-3 and CD200R3, CD200R4 and mouse MAIR-II, all of which contain a positively charged amino acid, such as lysine 
(K) or arginine (R), in their TM region (Takaki et al., 2006). In contrast, FcR $\gamma$ chain is also able to associate with receptors, such as Fc $\gamma$ RIIIA and FceRI, which do not contain a positively charged amino acid in the TM region (Kim et al., 2003)(Varin-Blank and Metzger, 1990). Because MAIR-IV does not possess a positively charged amino acid in the TM region, FcR $\gamma$ chain might be a partner of MAIR-IV. In fact, a recent report by Fujimoto et al showed that the transfection of FcR $\gamma$ chain enhanced cell surface expression of co-transfected Flag-tagged MAIR-IV/CLM-5 in HEK293T cells, suggesting that MAIR-IV/CLM5 might associate with FcR $\gamma$ chain (Fujimoto et al., 2006). To examine whether MAIR-IV physically associates with the FcR $\gamma$ chain, a macrophage cell line, RAW264, which expressed the endogeneous FcR $\gamma$ chain, was transfected with MAIR-IV or MAIR-V tagged with Flag. As demonstrated in Fig 3A, MAIR-IV, but not MAIR-V, was coimmunoprecipitated with FcR $\gamma$ chain. We also transfected a rat basophilic leukemia cell line, RBL-2H3, with DAP12 tagged with Flag. Whereas MAIR-II was coimmunoprecipitated with DAP12, as previously reported by our group (Yotsumoto et al., 2003)(Nakahashi et al., 2007), MAIR-IV was not (Fig 3B). These results indicated the physical association of transfected MAIR-IV with the FcR $\gamma$ 
chain, but not with DAP12, in these transfectants. Furthermore, we also found that endogeneous MAIR-IV was also coimmunoprecipitated with FcR $\gamma$ chain in lysates of peritoneal macrophages stimulated with thioglycollate from wild type (WT), but not $F_{C} R \gamma^{-/-}$, mice. In addition, cell surface expression of MAIR-IV was significantly down-regulated on bone marrow and peritoneal neutrophils from $F c R \gamma^{-/-}$mice, compared with those from WT mice (Fig. 3D). Moreover, although intraperitoneal treatment with thioglycollate significantly up-regulated cell surface expression of MAIR-IV on macrophages, this enhancement was not detected on those from $F_{c} R \gamma^{-/-}$ mice (Fig. 3D). Because mRNA expression of MAIR-IV in $F c R \gamma^{-/-}$peritoneal macrophages was comparable to that in WT peritoneal macrophages (data not shown), cell-surface expression of MAIR-IV largely depended on FcR $\gamma$ chain expression. Taken together, these results indicated that FcR $\gamma$ chain physically associates with MAIR-IV and is involved in cell surface expression of MAIR-IV in primary neutrophils and macrophages. 
Nakano T, et al

\subsection{MAIR-IV is a triggering receptor on neutrophils}

Because MAIR-IV associates with FcR $\gamma$ chain, it may mediates an activating signal in neutrophils. To address this issue, neutrophils were purified from the BM and peritoneal cavity and stimulated with plate-coated F(ab') 2 fragments of anti-MAIR-IV mAb. As shown in Fig. 4A, TNF- $\alpha$ secretion from neutrophils was significantly increased after the stimulation with anti-MAIR-IV. Similarly, stimulation of PEC with plate-coated F(ab’) 2 fragments of anti-MAIR-IV mAb significantly augmented secretion of IL-6 as well as TNF- $\alpha$ (Fig. 4B). These results indicated that MAIR-IV is a novel activating receptor on neutrophils. Thus, MAIR-IV may regulate neutophils activation and involved in innate immunity. To clarify the in vivo role of MAIR-IV in immune responses, identification of the ligand for MAIR-IV should be required.

\section{Acknowledgement}

This research was supported in part by the grants provided by the Ministry of Education, 
Nakano T, et al

Science and Culture of Japan. We thank Yurika Soeda for secretarial assistance. 


\section{REFERENCES}

Cantoni C., Bottino C., Augugliaro R., Morelli L., Marcenaro E., Castriconi R., Vitale M., Pende D., Sivori S., Millo R., Biassoni R., Moretta L. and Moretta A. (1999) Molecular and functional characterization of IRp60, a member of the immunoglobulin superfamily that functions as an inhibitory receptor in human NK cells. Eur J Immunol 29, 3148-59.

Chung D. H., Humphrey M. B., Nakamura M. C., Ginzinger D. G., Seaman W. E. and Daws M. R. (2003) CMRF-35-like molecule-1, a novel mouse myeloid receptor, can inhibit osteoclast formation. J Immunol 171, 6541-8.

Clark G. J., Fitzpatrick S., Kuo B., Modra C., Jamriska L. and Hart D. N. (2002) CMRF-35A, CMRF-35H: potential new CD. J Biol Regul Homeost Agents 16, 233-5.

Clark G. J., Green B. J. and Hart D. N. (2000) The CMRF-35H gene structure predicts for an independently expressed member of an ITIM/ITAM pair of molecules localized to human chromosome 17. Tissue Antigens 55, 101-9.

Feng J., Garrity D., Call M. E., Moffett H. and Wucherpfennig K. W. (2005) Convergence on a distinctive assembly mechanism by unrelated families of activating immune receptors. Immunity 22, 427-38.

Fujimoto M., Takatsu H. and Ohno H. (2006) CMRF-35-like molecule-5 constitutes novel paired receptors, with CMRF-35-like molecule-1, to transduce activation signal upon association with FcRgamma. Int Immunol 18, 1499-508.

Kim M. K., Huang Z. Y., Hwang P. H., Jones B. A., Sato N., Hunter S., Kim-Han T. H., Worth R. G., Indik Z. K. and Schreiber A. D. (2003) Fcgamma receptor transmembrane domains: role in cell surface expression, gamma chain interaction, and phagocytosis. Blood 101, 4479-84.

Kumagai H., Oki T., Tamitsu K., Feng S. Z., Ono M., Nakajima H., Bao Y. C., Kawakami Y., Nagayoshi K., Copeland N. G., Gilbert D. J., Jenkins N. A., Kawakami T. and Kitamura T. (2003) Identification and characterization of a new pair of immunoglobulin-like receptors LMIR1 and 2 derived from murine bone marrow-derived mast cells. Biochem Biophys Res Commun 307, 719-29.

Luo K., Zhang W., Sui L., Li N., Zhang M., Ma X., Zhang L. and Cao X. (2001) DIgR1, a novel membrane receptor of the immunoglobulin gene superfamily, is preferentially expressed by antigen-presenting cells. Biochem Biophys Res Commun 287, 35-41.

Nakahashi C., Tahara-Hanaoka S., Totsuka N., Okoshi Y., Takai T., Ohkohchi N., Honda S., Shibuya K. and Shibuya A. (2007) Dual assemblies of an activating immune receptor, MAIR-II, with ITAM-bearing adapters DAP12 and FcRgamma chain on peritoneal macrophages. J Immunol 178, 765-70.

Nathan C. (2006) Neutrophils and immunity: challenges and opportunities. Nat Rev Immunol 6, 173-82.

Shi L., Luo K., Xia D., Chen T., Chen G., Jiang Y., Li N. and Cao X. (2006) DIgR2, dendritic cell-derived immunoglobulin receptor 2 , is one representative of a family of IgSF inhibitory receptors and mediates negative regulation of dendritic cell-initiated antigen-specific T-cell responses. Blood 108, 2678-86.

Takaki R., Watson S. R. and Lanier L. L. (2006) DAP12: an adapter protein with dual 
functionality. Immunol Rev 214, 118-29.

Varin-Blank N. and Metzger H. (1990) Surface expression of mutated subunits of the high affinity mast cell receptor for IgE. J Biol Chem 265, 15685-94.

Yotsumoto K., Okoshi Y., Shibuya K., Yamazaki S., Tahara-Hanaoka S., Honda S., Osawa M., Kuroiwa A., Matsuda Y., Tenen D. G., Iwama A., Nakauchi H. and Shibuya A. (2003) Paired activating and inhibitory immunoglobulin-like receptors, MAIR-I and MAIR-II, regulate mast cell and macrophage activation. J Exp Med 198, 223-33. 
Figure Legends

Figure 1. Chromosomal localization and molecular phylogenetic tree of MAIR gene family.

(A) Chromosomal localization of mouse and human MAIR families on chromosome 11 and 17, respectively, were determined based on the data from National Center for Biotechnology Information Map Viewer site. (B) Phylogenetic analysis of the MAIR family genes was performed by using the UPGMA method of GENETYX-MAC software (Software develoment, Tokyo, Japan).

Figure 2. Expression of MAIR-IV on hematopoietic cells.

(A) BW5147 parent cells or transfectants expressing Flag-tagged MAIR proteins indicated were stained with anti-Flag mAb (open histgrams of the upper panel) or Alexa-647-conjugated F(ab’) 2 fragments of TX69 mAb (open histograms of the lower panel). Shaded histograms shows negative controls with isotype-matched control IgGs.

(B) Peripheral blood, spleen, bone marrow (BM), peritoneal exudative cells (PEC), 
BM-derived cultured macrophages, dendritic cells (DC) and mast cells from C57BL/6 mice were stained with Alexa-647-conjugated F(ab') 2 fragments of TX69 mAb (open histograms) or $\mathrm{F}(\mathrm{ab})_{2}$ fragments of isotype-matched control antibodies (shaded histograms) in combination with lineage-specific mAbs indicated. Cells were gated according to lineage markers expression by flow cytometry.

\section{Figure 3. MAIR-IV associates with FcR $\gamma$.}

(A) RAW264 cells or transfectants expressing Flag-tagged MAIR-IV or MAIR-V were lysed and immunoprecipitated with anti-Flag and proteins were immunoblotted with anti-Flag or anti-FcR $\gamma$. (B) RBL-2H3 cells were transfected with Flag-tagged DAP12 alone or in combination with MAIR-IV or MAIR-II. These transfectants were lysed and immunoprecipitated with anti-MAIR-IV (TX81) or anti-MAIR-II (TX10) mAbs and proteins were immunoblotted with anti-MAIR-IV (TX69) or anti-Flag mAb. (C) Macrophages were collected from peritoneal cavity of WT or FcR $\gamma$-/- mice 4 days after i.p. treatment with thioglycollate, lysed and immunoprecipitated with anti-MAIR-IV mAb (TX81). Immunoprecipitates were immunoblotted with anti-MAIR-IV (TX69) 
or anti-FcR $\gamma$ mAbs. (D) Neutrophils were purified from BM and peritoneal cavity from WT or $F c R \gamma$-/- mice 6 hrs after i.p. treatment with thioglycollate. Macrophages were collected from peritoneal cavity of WT or $F c R \gamma$-/- mice before or 4days after i.p. treatment with thioglycollate. Cells were stained with Alexa647-conjugated F(ab')2 fragments of anti-MAIR-IV (TX69), PE-conjugated anti-Gr-1, and FITC-conjugated anti-Mac-1 mAbs.

Figure 4. MAIR-IV triggers secretion of inflammatory cytokines from neutrophils macrophages.

$\mathrm{BM}$ and peritoneal neutrophils (A) and resident peritoneal macrophages (B) were stimulated with either plate-coated F(ab') 2 fragments of anti-MAIR-IV (TX69) or isotype-matched control mAbs for 12 hrs and 24 hrs, respectively, and TNF- $\alpha$ and IL-6 concentrations in the supernatants were measured by ELISA. * and ** indicate $\mathrm{p}<0.05$ and $\mathrm{p}<0.01$, respectively, as analysed by Student's $t$-test. Data are respresentative from three independent experiments. 
Figure 1

A

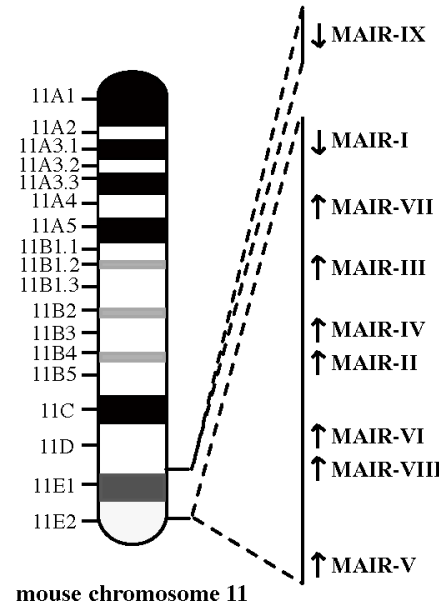

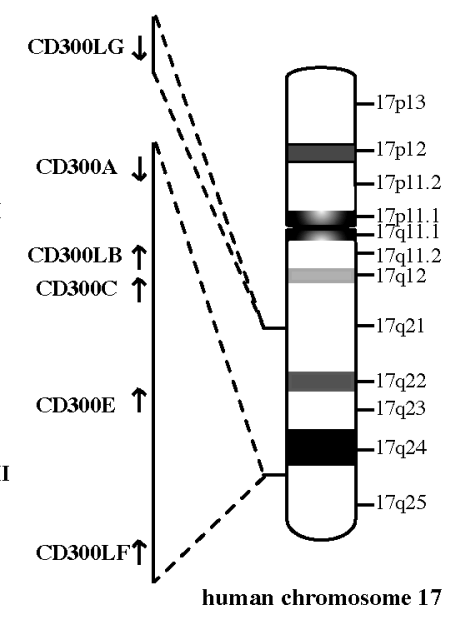

B

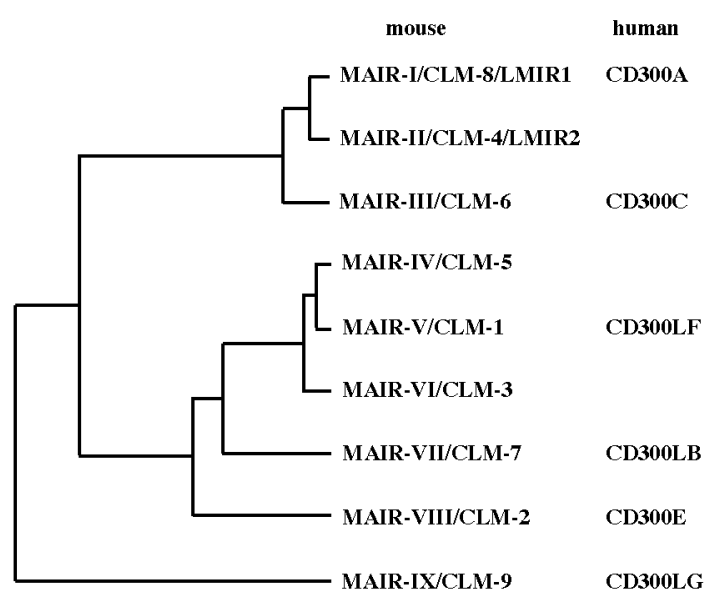


Figure 2

A
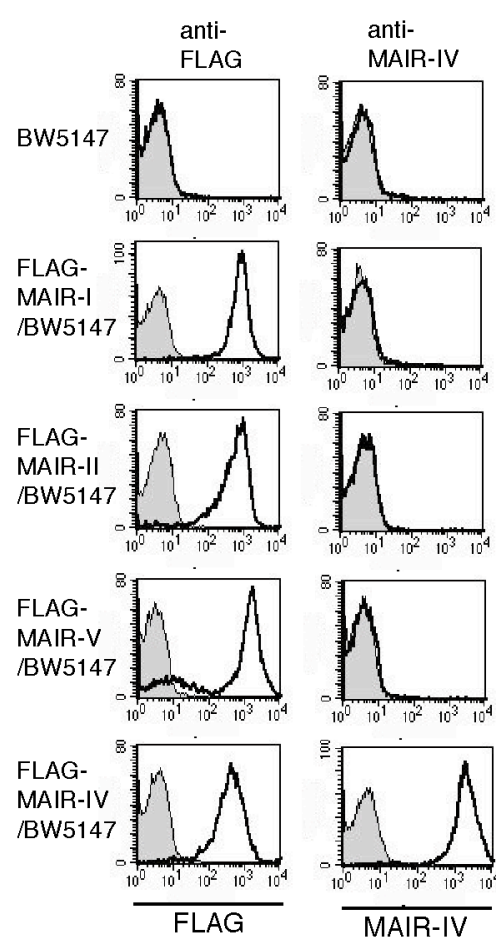
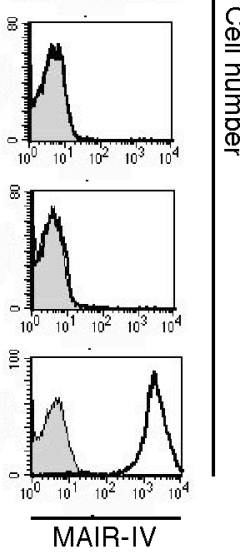

B
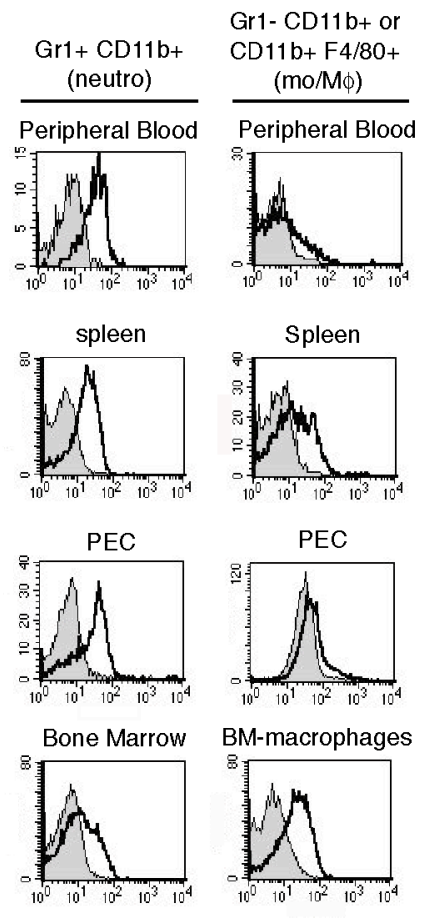
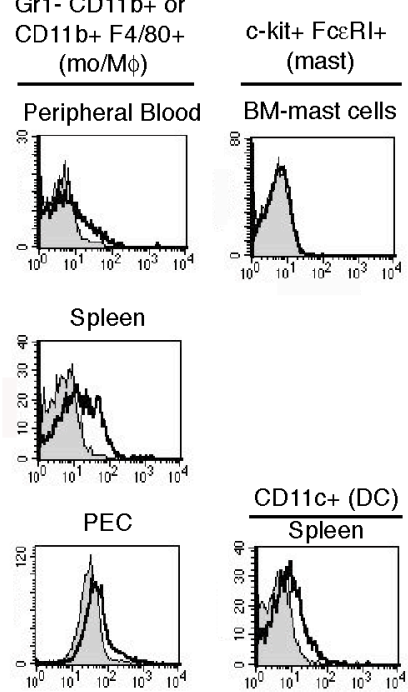

BM-macrophages

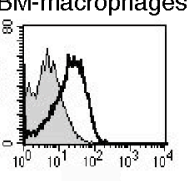

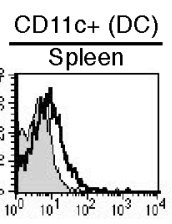

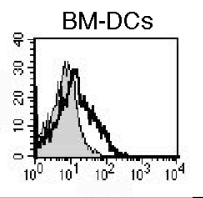

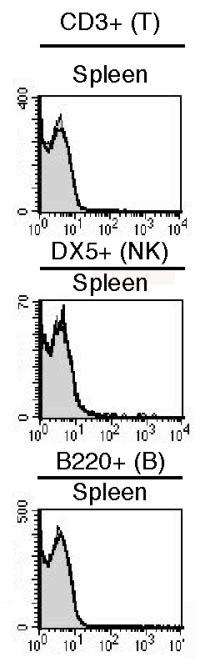

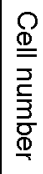

MAIR-IV 


\section{Figure 3}

A

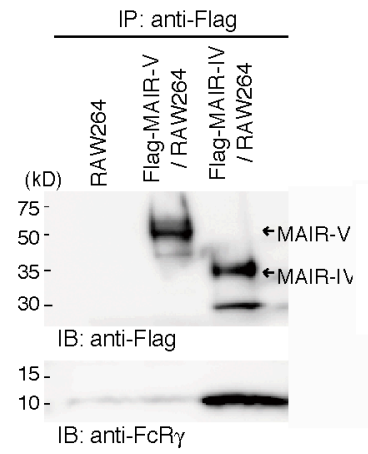

C

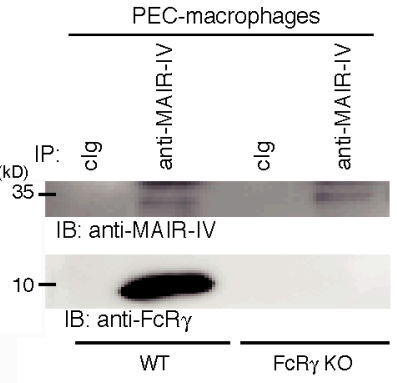

B

Flag-DAP12 Flag-DAP12 Flag-DAP12 Flag-DAP12

alone/RBL + MAIR-IV/RBL alone/RBL + MAIR-||/RBL
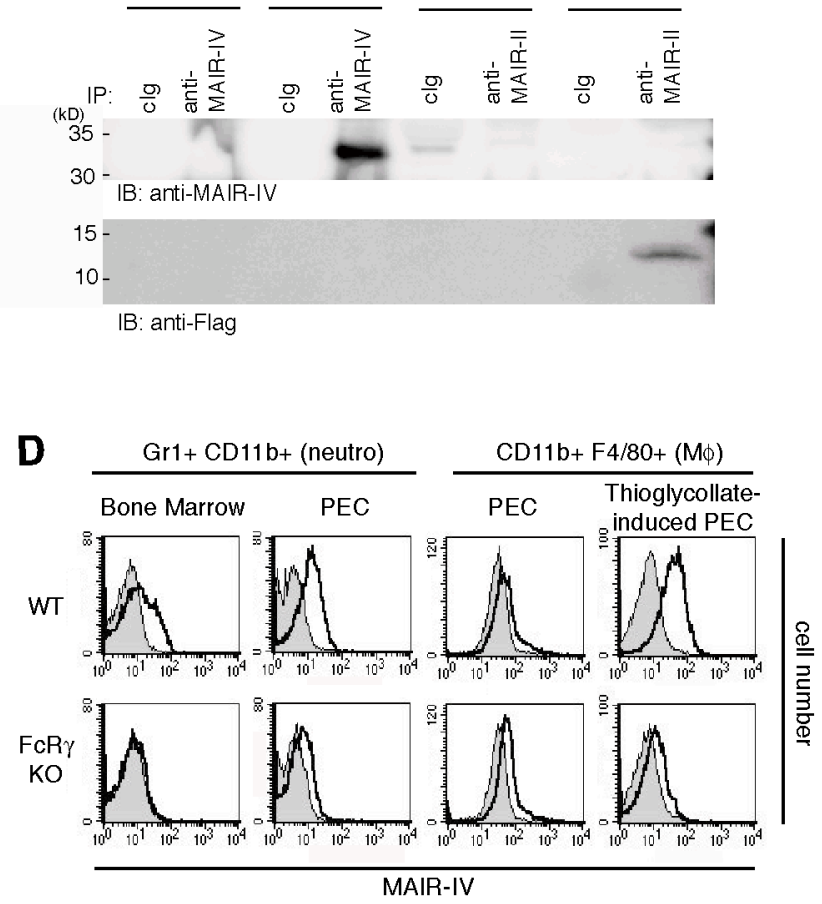
Figure 4

A

$\frac{\text { Gr1 + neutrophils }}{\text { BM derived } \quad \text { Thioglycollate-induced }}$

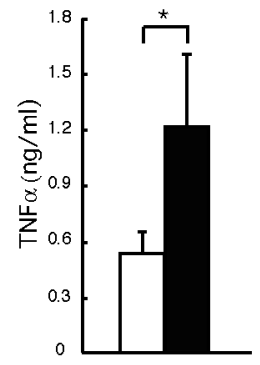

PEC derived

B
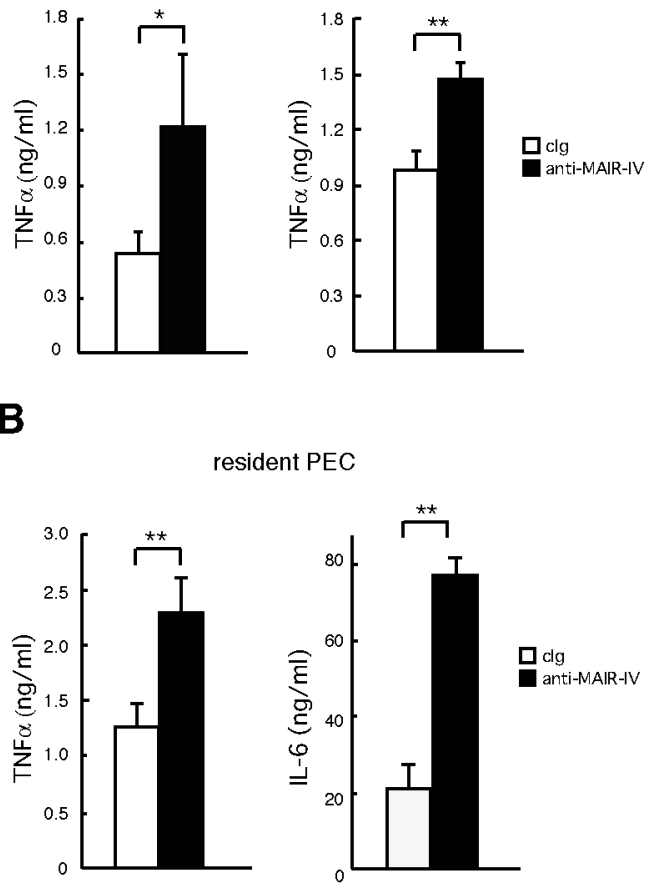